\title{
Libros de texto y planificación docente ante la enseñanza de la gramática en educación secundaria
}

Recepción: 23/07/2020 | Revisión: 23/09/2020 | Aceptación: 02/01/2021 | Preprint: 17/03/2021 | Publicación: 01/03/2022

\author{
Antonio GUTIÉRREZ RIVERO \\ Universidad de Cádiz \\ antoniogutierrez.rivero@uca.es \\ https://orcid.org/oooo-0oo1-6223-2390
}

\author{
Manuel Francisco ROMERO OLIVA \\ Universidad de Cádiz \\ manuelfrancisco.romero@uca.es \\ https://orcid.org/oooo-00o2-6854-0682
}

\begin{abstract}
Resumen: Esta investigación analiza la contribución de los libros de texto a la enseñanza de la lengua en cuarto curso de educación secundaria desde la planificación de los docentes. Se ha realizado una triangulación de los elementos principales de los procesos de enseñanza-aprendizaje: a) el profesorado, como agente clave en los procesos de enseñanza/aprendizaje; $b$ ) el alumnado, como destinatario y protagonista del proceso; c) el libro de texto, como recurso nuclear que pervive actualmente en el aula. Los objetivos principales de la investigación son el análisis de la transposición didáctica llevada a cabo por los docentes y los libros de texto, y la valoración de la competencia gramatical de los alumnos. Este artículo se centra en los docentes y los libros de texto. Los resultados demuestran la necesidad de un cambio en los planteamientos metodológicos de los libros de texto y en la dinamización de estos recursos en el aula por parte de los docentes. Ambos siguen instalados en metodologías que no promueven la reflexión lingüística y perpetúan un modelo basado en la memorización de los contenidos, exenta de razonamiento. Por ello, se propone un cambio metodológico con la unidad textual como punto de partida para la reflexión gramatical.
\end{abstract}

Palabras clave: enseñanza de la lengua; competencia gramatical; planificación docente; libros de texto.

\section{TEXTBOOKS AND TEACHING PLANNING CONCERNING TEACHING GRAMMAR IN SECONDARY EDUCATION}

\begin{abstract}
This research analyzes the contribution of textbooks to language teaching in the fourth year of secondary education from the point of view of teaching planning. The key elements of the teaching-learning processes have been triangulated: a) teachers, as key agents in teaching-learning processes; $b$ ) students, as receivers and protagonists of the process; c) textbooks, as main resource that survives in the classroom. The main objectives of the research are the analysis of didactic transposition carried out by teachers and textbooks and the assessment of grammar competence of the students. This academic paper focuses on the teaching staff and textbooks. The results show that a change is currently needed in the methodological approaches to textbooks as well as in the dynamization of these resources in the classroom by the teachers. Both textbooks and teachers utilize methodologies that do not promote linguistic reflection and perpetuate a model based on memorization of contents, exempt of reasoning. Thus, a methodological change is proposed with the textual unit as a starting point for grammatical reflection.
\end{abstract}

Keywords: language teaching; grammatical competence; teaching planning; textbooks. 


\section{LLIBRES DE TEXT I PLANIFICACIÓ DOCENT DE L'ENSENYAMENT DE LA GRAMÀTICA EN EDUCACIÓ SECUNDÀRIA}

Resum: Aquesta recerca analitza la contribució dels llibres de text a l'ensenyament de la lengua a quart curs d'educació secundària des de la planificació dels docents. S'ha realitzat una triangulació dels elements principals dels processos d'ensenyament-aprenentatge: a) el professorat, com agent clau en els processos d'ensenyament-aprenentatge; b) l'alumnat, com destinatari i protagonista del procés; c) el llibre de text, com recurs nuclear que perviu actualment a l'aula. Els objectius principals de la recerca són l'anàlisi de la transposició didàctica duta a terme pels docents i els llibres de text, $i$ la valoració de la competència gramatical dels alumnes. Aquest article se centra en els docents i els llibres de text. Els resultats mostren la necessitat d'un canvi en els plantejaments metodològics dels llibres de text $i$ en la dinamització d'aquests recursos a l'aula per part dels docents. Ambdós segueixen instal-lats en metodologies que no promouen la reflexió lingüística i perpetuen un model basat en la memorització dels continguts, exempta de raonament. Per tant, es proposa un canvi metodològic amb la unitat textual com punt de partida per a la reflexió gramatical.

Paraules clau: ensenyament de la llengua; competència gramatical; planificació docent; llibres de text.

\section{Introducción}

El propósito fundamental de esta investigación es aportar datos empíricos que corroboren la hipótesis de la pervivencia de una cultura de aprendizaje basada en el conocimiento y la clasificación de las formas lingüísticas, exento de toda reflexión significativa en la enseñanza de la lengua. Nos centraremos para este estudio en la etapa de Educación Secundaria Obligatoria (en adelante, ESO). Consideramos que hay una concepción heredada de la formación recibida por los propios profesores en las etapas conducentes a la función docente, unida a una dependencia del libro de texto, tan criticado y rechazado desde los movimientos de renovación pedagógica, pero que se mantiene como piedra angular en los procesos de enseñanza-aprendizaje (Gutiérrez y Gaviño, 2020; Jiménez Fernández, 2011).

Desde este panorama inicial, planteamos una investigación donde se integren los propios agentes de la práctica educativa (Chevallard, 1985) -docentes, estudiantes y materiales pedagógicos-, partiendo de los condicionantes culturales que han impregnado la práctica docente en la enseñanza de la lengua en nuestro país, ya que el tipo de transposición didáctica elegida determina en gran medida la labor docente (Álvarez Angulo, 2013). Nuestro estudio pretende ir más allá de los puros conceptos teóricos y epistemológicos de las disciplinas lingüísticas, e intervenir "en la identificación de los problemas relacionados con la enseñanza y aprendizaje de la lengua y la literatura, y en presentar propuestas válidas mediante la investigación en la acción para solucionarlos, a partir de los condicionantes implicados en el proceso didáctico" (Prado, 2011, p. 34). En la Figura 1 se resume la relación entre los agentes que intervienen en nuestra investigación: por un lado, los docentes y su concepción de la enseñanza de la lengua y los materiales que estos utilizan (el libro de texto en concreto) y el enfoque que adoptan para la transposición didáctica; y por otro, los alumnos en los que se analizó su competencia gramatical y, también, su concepción sobre el estudio de la lengua. 


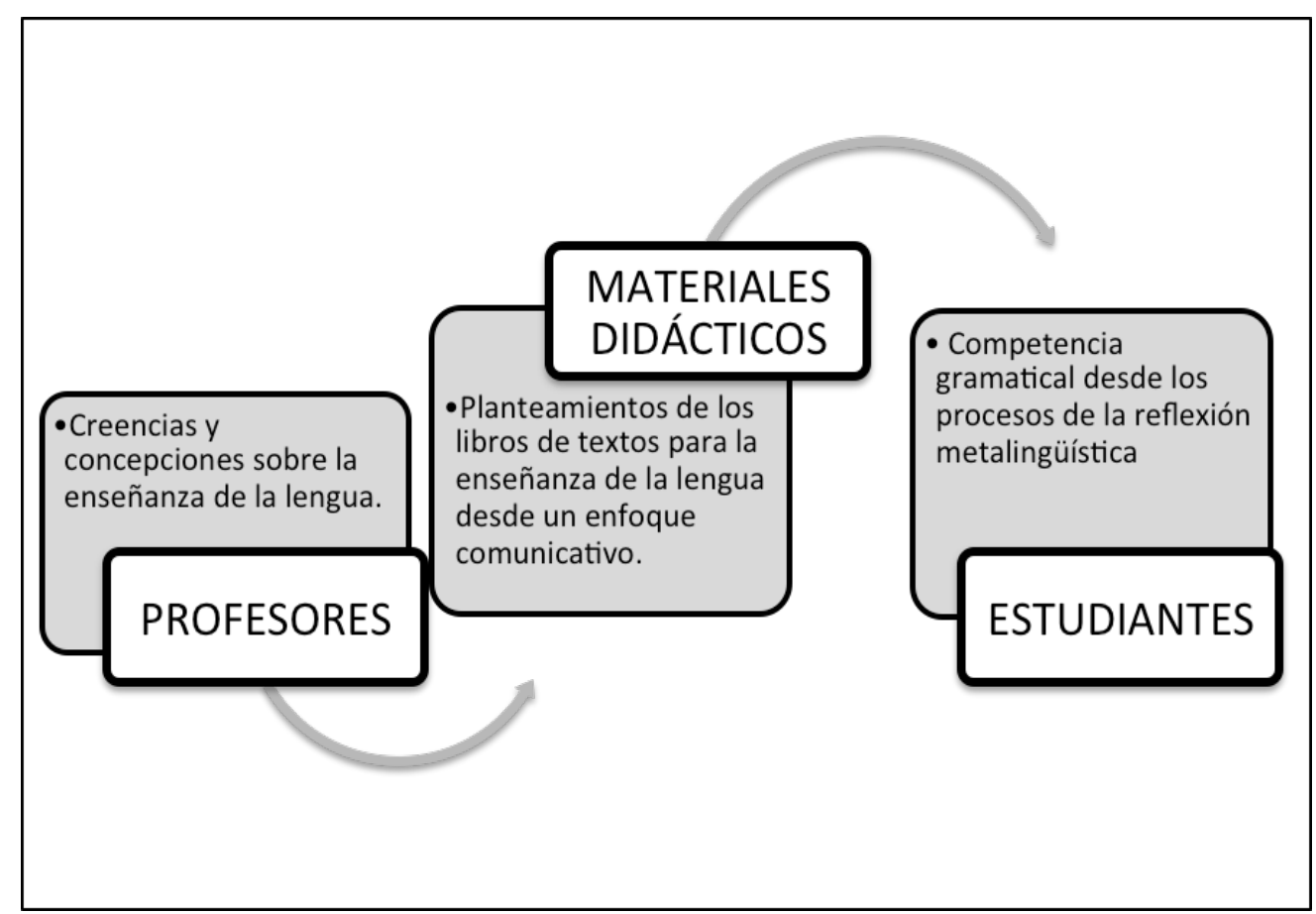

Figura 1. Transposición didáctica y sujetos de la investigación. Fuente: Propia.

Esta investigación se integra en una de carácter superior que tiene como centro de interés indagar sobre el papel de la gramática en la enseñanza de la lengua y que se detalla en dos objetivos específicos: en primer lugar, analizar la cultura de enseñanza entre docentes de educación secundaria (planificación del profesorado respecto a la enseñanza de la lengua y el material que emplean, fundamentalmente, los libros de texto); y, posteriormente, determinar los niveles de adquisición y dominio de la lengua en relación con la competencia gramatical de una muestra de estudiantes de $4 .^{\circ}$ de ESO. En este artículo, por cuestiones de espacio, nos centramos en el primer objetivo, mientras que los referidos al segundo, se convierten en objeto de transferencia en otras publicaciones.

\section{La enseñanza de la lengua desde la tradición educativa}

El contexto educativo actual en España es el resultado de casi 200 años de ordenación de la instrucción pública, aderezado por la convulsa historia del país durante la era moderna. Desde el siglo XIX se empezaron a regular los sistemas educativos nacionales en Europa, lo que tuvo una repercusión en los primeros intentos de trasladar esa situación a España bajo la influencia de las Cortes de Cádiz. No obstante, no sería hasta 1857 con la Ley de Instrucción pública, la llamada Ley Moyano, cuando se ordenaría por primera vez la educación a nivel nacional. Dicha ley permaneció vigente (aunque con pequeñas reformas) hasta el final del siglo XX al llevarse a cabo la segunda gran reforma, la Ley General de Educación (1970) o Ley Villar Palasí. Bajo los postulados de la primera llegaron a estudiar los españoles nacidos antes de los años 70, y bajo la segunda se han formado los nacidos hasta los 80 . Ambos grupos de edad constituyen una buena parte de los profesionales todavía en activo entre el profesorado de nuestros centros educativos. 
En 1990 se enmarca el tercer gran hito en la evolución del sistema educativo español con la promulgación de la Ley Orgánica de Ordenación General del Sistema Educativo (LOGSE) que supone un cambio de perspectiva en la filosofía de la normativa en materia educativa. Se introduce un conjunto de presupuestos que modifica en gran medida la concepción del proceso de enseñanza-aprendizaje y del funcionamiento de los centros. Entre otros: una base pedagógica fundamentada en los principios del constructivismo, que introduce un cambio de perspectiva al colocar al alumno en el centro del proceso de aprendizaje $y$, en consecuencia, otorgar un papel menos central al docente que se convierte así en un planificador, guía o coordinador de ese proceso; un currículo más flexible basado en niveles de concreción para otorgar más autonomía a los centros; y la introducción del aprendizaje por competencias que trata de superar la condición de las materias como compartimentos estancos y conectar los saberes aprendidos en la escuela con la vida real. Las leyes posteriores (LOCE [2002], LOE [2006], LOMCE [2013]) no han introducido cambios relevantes a los establecidos en la reforma de los años 90.

Esta concepción de la educación difería en gran medida de la que estaba presente en la escuela hasta entonces y en lo que respecta a la enseñanza de la lengua propiciaba un escenario favorable al enfoque comunicativo. Lógicamente, ello no implica obviar la gramática ya que "la reflexión gramatical deberá considerarse como un componente adicional del aprendizaje de la lengua" (Martínez Montes et al., 2015, p. 82). Además, la gramática es fundamental para comprender la estructuración de los elementos como parte del conjunto. Tan negativo es que el aprendizaje de una lengua no incluya el aprendizaje gramatical, como que se quiera convertir la clase de lengua en clase de lingüística (González Nieto, 2001), lo que nos hace recordar las palabras de Chomsky: "hemos confundido ciencia con educación" (citado en Castellà, 1994, p. 17) y que acentúan el protagonismo que ha tenido la gramática aislada del uso en nuestras aulas.

Sin embargo, en lo que concierne a la enseñanza de la lengua, la herencia de los viejos planes de estudio se refleja en una pedagogía centrada en la gramática. Esta forma de enseñar la lengua tiene al profesor y al libro como vehículos de transmisión de unos conocimientos que son generalmente memorizados por los alumnos sin la necesaria reflexión sobre los significados y los usos, lo que provoca un efecto no deseado desde la filosofia de la didáctica y leyes educativas actuales:

\begin{abstract}
Los alumnos acaban identificando la lengua con el libro de texto o la gramática con una asignatura escolar. Están muy lejos de darse cuenta y de entender que la lengua es la que utilizan cada día para comunicarse, jugar, hablar, estudiar, aprender, etc.; que con la lengua pueden leer historias divertidas, inventar personajes o explicar chistes con gracia. (Cassany, Luna, y Sanz, 1994, pp. 14-15)
\end{abstract}

Por ello, uno de los objetivos de este trabajo se centra en indagar si, pese al supuesto cambio metodológico propuesto desde el currículo, esa concepción tradicional de la enseñanza de la lengua sigue vigente en el profesorado, como dinamizadores del proceso de enseñanzaaprendizaje en la escuela, y patente en los libros de texto, como material de referencia en los procesos de aprendizaje. Pues, como indica Jiménez Fernández:

A pesar de que el libro de texto ha recibido en las últimas décadas una crítica acérrima por parte de divdersos movimientos de renovación pedagógica, la verdad es que aún sigue siendo una herramienta omnipresente en la enseñanza obligatoria. (Jiménez Fernández, 2011) 
En el libro se aúnan la cultura escolar y los valores imperantes en la sociedad de cada momento. Cumple tres funciones: es el soporte curricular, el espejo donde se refleja la sociedad que lo produce y el espacio de memoria de los métodos de enseñanza-aprendizaje utilizados (Escolano, 1997).

Aun así, cabe preguntarse qué es el libro de texto y de qué manera se puede considerar como representativo de los medios de enseñanza que se utilizan en las clases (Johnsen, 1996). Hoy en día, con la revolución digital los profesores tienen a su disposición mucha información para complementar u organizar sus clases. Incluso el no utilizarlo nos puede llevar a afirmar que, "Los libros controlan la situación del aula hasta el punto de que no usarlos se considera como una muestra de independencia" (Johansson, 1988, p. 35). Sin embargo, la mayoría de docentes, los de esta investigación también, lo utilizan como herramienta fundamental para sus clases siguiendo la creencia extendida de que la programación del aula gira en torno a él (Ferrer, 2015, p. 52).

Los estudios apuntan a que el modelo tradicional basado en la memorización de contenidos, el reconocimiento y la clasificación de unidades gramaticales descontextualizadas no contribuye a mejorar los usos lingüísticos de los alumnos (Jiménez Fernández, 2011), por lo que es necesario un enfoque gramatical más funcional (Martín Vegas, 2009), basado en la relación de los contenidos gramaticales, semánticos y pragmáticos y en el fomento de la actividad de hablar del alumno para favorecer la conexión entre la reflexión lingüística y el uso (Camps, 2017). Dicho modelo tradicional se basa en las corrientes lingüísticas anteriores a la Lingüística del Texto y tiene como objeto la identificación de categorías gramaticales y sus funciones, por lo que no se vincula con un aprendizaje basado en el propio uso de la lengua (Zayas, 2006; 2011). La transposición didáctica debería plasmarse en metodologías que conviertan a los estudiantes en usuarios competentes de la lengua y no en el trasvase de teorías lingüísticas a la escuela sin relación con el uso (Castellà, 1994). Por tanto, es necesaria una metodología que encamine al alumno en la clase de lengua a aprender a "saber hacer cosas con las palabras" (Austin, 1962), donde la mediación del docente es básica en lo que respecta a la selección y dinamización de los recursos didácticos, específicamente de los libros de texto. El rol del docente debe centrarse en la planificación del proceso de enseñanza-aprendizaje, creando situaciones comunicativas que promuevan la reflexión consciente por parte del alumnado a través de la observación y manipulación de enunciados. En el costoso (cognitivamente) proceso de abstracción que debe realizar el alumno el docente tiene que acompañarlo, anticipando problemas y solvéntandolos a medida que van apareciendo. El libro de texto debe ser un aliado en ese proceso y favorecer metodologías que promuevan la reflexión lingüística y no la memorización de contenidos sin sentido.

Esto nos lleva a preguntarnos hasta qué punto la pervivencia de ese modelo que podemos llamar tradicional se puede deber a la acción del libro de texto o del profesor, o de ambos; y al mismo tiempo, si el libro de texto como elemento angular en las clases puede llegar a ser el catalizador del cambio necesario en la enseñanza de la lengua. 


\section{Objetivos y metodología de la investigación}

Nuestra hipótesis de partida está centrada en que, a pesar de los cambios metodológicos en las leyes educativas de los útimos treinta años, se continúan aplicando planteamientos anteriores a dichas reformas. Dicho de otra manera, sigue vigente un modelo centrado en la enseñanza de la gramática, basado en la lingüística tradicional, los modelos estructuralistas y generativos que prioriza el aprendizaje mecanicista, taxonomista, centrado en la forma y no en el significado. En consecuencia, no propicia un desarrollo pleno de las habilidades comunicativas, a causa de una cultura de los procesos de enseñanza-aprendizaje heredados de los docentes que perpetúa un modelo basado en la gramática.

Esta primera consideración, como hemos venido anticipando, determina el propósito de nuestro estudio inicial, al pretender revisar la aportación de la enseñanza de la gramática a la adquisición de la competencia en comunicación lingüística en cuarto curso de ESO. Para ello, se atenderá a la triangulación de los siguientes elementos: a) el profesorado, como agente clave en los procesos de enseñanza/aprendizaje; $b$ ) el alumnado, como destinatario y protagonista del proceso; $y, c)$ el libro de texto, como recurso nuclear que pervive actualmente en el aula.

Los objetivos, sujetos e instrumentos de la investigación quedan recogidos a continuación (Tabla 1):

\begin{tabular}{lcc}
\hline \multicolumn{1}{c}{ Objetivos } & Sujetos & Instrumentos \\
\hline $\begin{array}{l}\text { Analizar la planificación de los docentes de Lengua } \\
\text { respecto a su práctica docente en cuarto curso de ESO. }\end{array}$ & Profesores & Cuestionario \\
$\begin{array}{l}\text { Comprobar el nivel de concreción de los libros de texto a } \\
\text { las prescripciones del currículo en relación con la }\end{array}$ & Libros de & Rúbrica de \\
competencia en comunicación lingǘstica. & valoración \\
$\begin{array}{l}\text { Determinar el nivel de dominio gramatical de los alumnos } \\
\text { de cuarto curso de ESO en el análisis sintáctico y }\end{array}$ & Estudiantes & Prueba de \\
semántico de oraciones. & & gramatical \\
\hline
\end{tabular}

Tabla 1. Objetivos, sujetos e instrumentos de la investigación.

Como hemos señalado anteriormente, nuestro análisis aquí se centra en los dos primeros sujetos.

\subsection{Muestra de estudio}

Todo estudio requiere la delimitación de unas coordenadas de espacio y tiempo; en nuestro caso, la población de estudio se centró en los estudiantes de $4 .{ }^{\circ}$ curso de ESO de la ciudad de SeviIla, al ser una de las aglomeraciones urbanas más importantes de España y la más importante de la comunidad autónoma andaluza. Por tanto, constituye una muestra representativa para nuestro estudio y extrapolable a un contexto geográfico mayor. Sobre una población de 5.971 
alumnos matriculados en $4 .{ }^{\circ}$ de ESO, el tamaño muestral es de $447(n)$, de lo que podemos verificar que el nivel de confianza ( $1-a)$ es superior al $95 \%$, con un parámetro $(p=0.5)$ que lo maximiza. Se puede observar cómo se distribuyó la población de estudio (Tabla 2 ):

\begin{tabular}{clc}
\hline Sujetos & \multicolumn{1}{c}{ Distribución } & Total \\
\hline Estudiantes & Centros públicos (11) & \\
& $n 253$ & \\
& Centros privados-concertados (6) & $n 447$ \\
& $n 119$ & \\
& Centros privados (3) & \\
& $n 75$ \\
Profesores & Centros públicos (11) & \\
& $n 11$ & \\
& Centros privados-concertados (6) & \\
& $n 6$ \\
& Centros privados (3) & \\
& $n 3$ \\
& Editoriales & $n 10$ \\
& $n 10$ & \\
\hline
\end{tabular}

Tabla 2. Objetivos, sujetos e instrumentos de la investigación.

\subsection{Metodología}

La metodología empleada en nuestro estudio, tomando como referencia a McMillan y Schumacher (2005) se encuadra en una modalidad de investigación híbrida, cualitativa y cuantitativa, que augura una investigación interpretativa:

- por una parte, llevamos a cabo un estudio de campo donde empleamos técnicas de recogida de datos de ambos métodos: a) análisis documental, b) cuestionarios, y c) entrevistas.

- por otra parte, el análisis de los resultados será de índole cuantitativa no experimental descriptiva, ya que nuestro propósito es averiguar si el déficit que presenta el alumnado de ESO en el ámbito de la gramática viene determinado por una enseñanza descontextualizada de la morfosintaxis y poco vinculada a los contenidos semánticos. Para ello, describiremos y evaluaremos las condiciones en que se produce dicho proceso y los factores que en él intervienen (profesorado y materiales didácticos). El fin es establecer una correlación entre los planteamientos docentes y los materiales que utilizan, y los procesos de enseñanza y aprendizaje que verifique si las causas que lo originan se asemejan a las planteadas previamente en nuestras hipótesis. 
Las diferentes actuaciones quedarán correlacionadas a través de la triangulación de los elementos de nuestra investigación como queda plasmado a continuación (Figura 2):

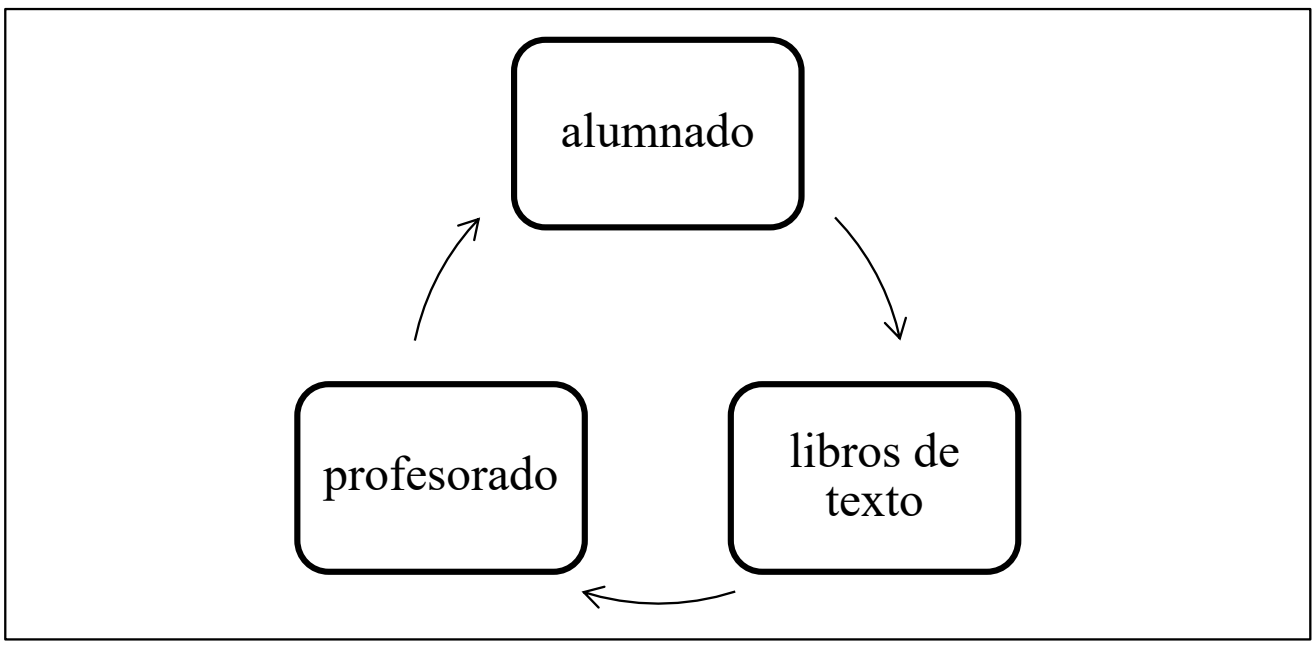

Figura 2. Triangulación metodológica de la investigación.

\subsection{Diseño y justificación de los instrumentos de la investigación}

Por limitaciones en el espacio de este artículo se presentan los instrumentos con sus resultados en el apartado 3.

\subsubsection{Cuestionario del profesorado: creencias docentes}

El objetivo del cuestionario realizado al profesorado consistió en definir el perfil académico y profesional de los informantes de la muestra a través de sus aportaciones sobre su visión de la docencia. Este se divide en tres bloques:

a) Planificación de la docencia: formado por dos preguntas sobre el orden de preferencia de criterios en la planificación de la clase, y la importancia otorgada al propio proceso de planificación. El objetivo era investigar si el profesorado organiza sus clases en función de los criterios ya dados (guías didácticas, diseño curricular base) de forma autónma, o si prefiere planificar de forma coordinada y en equipo.

b) Enfoques de la enseñanza: formado por cuatro preguntas sobre las preferencias acerca de los objetivos lingüísticos para enseñar y de las tipologías de ejercicios. El objetivo era descubrir a qué perfil responden los docentes en cuanto a cultura de enseñanza.

c) Aplicación de la docencia: formado por doce ítems referidos a la manera en que desarrollan la clase de lengua que los profesores debían valorar numéricamente. Se trataba de aunar los contenidos lingüísticos presentes en el currículo (Junta de Andalucía, 2007) y verificar si los docentes los llevan al aula. 
La validación de este cuestionario se llevó a cabo mediante tres procesos: la consulta a especialistas del ámbito de la didáctica de la lengua y la literatura (por un lado, de manera interna, entre miembros del departamento donde se adscribe la investigación; y, desde una perspectiva externa, mediante especialistas de didáctica de la lengua y la literatura de otras universidades y profesores de enseñanza secundaria de centros públicos y privados); en segundo lugar, se consultó a expertos del área de Didáctica y Organización Escolar (DOE) que, además de aportar su visión en el diseño de las pruebas, verificaron la adecuación en el contexto; y, finalmente, se realizó un cuestionario piloto para asegurar la relevancia de los resultados. Estas pruebas y las modificaciones resultantes verificaron la fiabilidad y validez del cuestionario.

\subsubsection{Rúbrica de evaluación de los libros de texto: paradigma formal versus funcional}

La rúbrica de los libros de texto utilizados por los profesores de la muestra se compone de dos partes ${ }^{\mathbf{1}}$ :

- En la primera de ellas, se atiende a datos técnicos que sirven para identificar y catalogar cada libro (titulo, editorial, autor/es, año de edición, soporte, número de páginas, ISBN).

- La segunda parte de la rúbrica corresponde a los ítems que tratan de verificar que los objetivos marcados en nuestro estudio se reflejan en los libros. Se trata de veintiocho enunciados que debían valorarse en una escala de Lickert del 1 al 5 en orden de consecución del objetivo.

La validación de esta rúbrica se realizó al igual que el resto de cuestionarios, siguiendo el mismo procedimiento. El proceso consistió en aplicar la escala de valoración por parte de varios investigadores. La relación entre las respuestas de los informantes que actuaron de jueces para la validación de la escala de valoración (Tabla 3) confirmó que la correlación estudiada era significativa al nivel 0,01 (bilateral).

\begin{tabular}{|c|c|c|c|c|}
\hline & & VAR00001 & VAR00002 & VAR00003 \\
\hline \multirow{3}{*}{$\begin{array}{l}\text { VAR0000 } \\
1\end{array}$} & Correlación de Pearson & 1 &, $681^{* *}$ &, $765^{* *}$ \\
\hline & Sig. (bilateral) & & ,000 &, 000 \\
\hline & $\mathrm{N}$ & 28 & 28 & 28 \\
\hline \multirow{3}{*}{$\begin{array}{l}\text { VAR0000 } \\
2\end{array}$} & Correlación de Pearson &, $681^{* *}$ & 1 &, $532^{* *}$ \\
\hline & Sig. (bilateral) &, 000 & &, 004 \\
\hline & $\mathrm{N}$ & 28 & 28 & 28 \\
\hline \multirow{3}{*}{$\begin{array}{l}\text { VAR0000 } \\
3\end{array}$} & Correlación de Pearson &, $765^{* *}$ &, $532^{* *}$ & 1 \\
\hline & Sig. (bilateral) &, 000 &, 004 & \\
\hline & $\mathrm{N}$ & 28 & 28 & 28 \\
\hline
\end{tabular}

\footnotetext{
${ }^{1}$ Los manuales analizados son: Alsina, R. et al. (2008), Andrés, P. et al. (2011), Bazarra, L. y Casanova, O. (2011), Gómez et al. (2011), González, A. (Coord.), Ariza et al. (2008), Juan, E. (dir.) et al. (2011), López, F. y Serrano, S. (2008), Navarro, E. y Reina, A. (2011) y VVAA (2008).
} 


\section{Hallazgos y análisis de los cuestionarios 3.1 En torno al cuestionario del profesorado}

El estudio de los resultados de cada bloque y los datos más relevantes surgidos de dicho análisis se concreta de la siguiente manera:

a) Respecto a la planificación de la docencia

Los docentes tienen una mayor preferencia en colegiar la planificación con otros colegas de su misma área y no tanto con los de otras áreas, lo que se aleja de una perspectiva interdisciplinar que sería muy adecuada para una enseñanza globalizadora en la que trabajar la lengua en diferentes áreas del currículo (Tabla 4):

1.1. Indique aquellos aspectos que intervienen a la hora de planificar su docencia siendo 1 nunca y 5 siempre:

\begin{tabular}{|l|c|}
\hline \multicolumn{1}{|c|}{ Enunciado } & Valoración \\
\hline 1.1.1. Programaciones y guías didácticas de las editoriales. & 3,4 \\
\hline 1.1.2. Propuestas del ministerio/consejería de educación & 4 \\
\hline 1.1.3. Acuerdos del equipo técnico. & 4,5 \\
\hline $\begin{array}{l}\text { 1.1.4. Equipos docentes de lengua para planificar la progresión de contenidos } \\
\text { en los cursos. }\end{array}$ & 4,45 \\
\hline $\begin{array}{l}\text { 1.1.5. Equipos docentes con otras asignaturas para trabajar la } \\
\text { interdisciplinariedad de los contenidos. }\end{array}$ & 3,3 \\
\hline
\end{tabular}

Tabla 4. Aspectos de planificación de docencia.

b) Respecto a los enfoques de la enseñanza de la lengua

De la comparación de los resultados de las respuestas del ítem 2.1. (Tabla 5) se observa que el enunciado que presenta mayor preferencia es "Saber comprender y expresar mensajes de forma adecuada, cohesionada, coherente y eficaz", que evidencia una disposición de los profesores a fomentar el desarrollo de las cuatro destrezas. Por otra parte, "Conocer el sistema formal de la lengua" no es uno de los ítems más elegidos por estos profesores. Ambos datos parecen indicar una preferencia por una enseñanza alejada de lo gramatical. Sin embargo, los profesores otorgaron menos importancia a "Conocer las variedades que tienen lugar en el uso de la lengua" que denota que, pese a su preferencia por el desarrollo de las habilidades lingüísticas, declaran menor interés por otra cuestión también relacionada con la lengua en uso.

2.1. Preferencias a la hora de enseñar lengua:

\begin{tabular}{|l|c|}
\hline \multicolumn{1}{|c|}{ Enunciado } & Valoración \\
\hline 2.1.1. Conocer el sistema formal de la lengua. & 3 \\
\hline 2.1.2. Mejorar su competencia textual. & 3,2 \\
\hline 2.1.3. Saber utilizar diversas estrategias y recursos para comunicar con eficacia & 3,3 \\
\hline 2.1.4. Conocer las variedades que tienen lugar en el uso de la lengua. & 1,9 \\
\hline $\begin{array}{l}\text { 2.1.5. Saber comprender y expresar mensajes de forma adecuada, cohesionada, } \\
\text { coherente y eficaz. }\end{array}$ & 4,35 \\
\hline
\end{tabular}

Tabla 5. Preferencias en la enseñanza de lenguas. 
Si comparamos esos datos con los del apartado 2.2 (Tabla 6), donde el mayor número de respuestas afirmativas correspondió a los tipos de ejercicios "Identificar y reconocer funciones sintácticas" (frecuencia de uso del 95\%) y "Localizar e identificar las categorías gramaticales" (90\% de frecuencia) se observa una evidente contradicción al optar por una tipología de ejercicios que podríamos llamar "tradicionales", basados en una aplicación mecánica de los contenidos. Se trata de ejercicios en los que hay realizar actividades como identificar, sustituir, completar, clasificar, rellenar espacios en blanco... (Jiménez Fernández, 2011), y que se oponen a otros en los que el alumno debe comparar, componer textos, recomponer, cambiar de orden, elidir... Estos últimos conllevan una construcción conjunta del conocimiento. Sin embargo, y nuevamente, el otro tipo de ejercicio basado en una concepción formal del aprendizaje, "Completar huecos en estructuras gramaticales" presenta muy poca preferencia, al igual que "Aplicar nuestras estructuras gramaticales para el dominio de otras lenguas".

2.2. Tipos de actividades para trabajar la gramática:

\begin{tabular}{|c|c|c|}
\hline Actividad & \multicolumn{2}{|c|}{$\begin{array}{c}\text { La utilizo } \\
\text { Sí/No }\end{array}$} \\
\hline 2.2.1. Localizar e identificar categorías gramaticales. & $90 \%$ & $10 \%$ \\
\hline 2.2.2. Completar huecos en estructuras gramaticales. & $30 \%$ & $70 \%$ \\
\hline 2.2.3. Identificar y reconocer funciones sintácticas. & $95 \%$ & $5 \%$ \\
\hline 2.2.4. Crear estructuras a partir de modelos dados. & $75 \%$ & $25 \%$ \\
\hline $\begin{array}{l}\text { 2.2.5. Aplicar conocimientos gramaticales para el desarrollo de la } \\
\text { comprensión. }\end{array}$ & $65 \%$ & $35 \%$ \\
\hline $\begin{array}{l}\text { 2.2.6. Ampliar y dominar el desarrollo textual a través de sus estructuras } \\
\text { gramaticales específicas. }\end{array}$ & $70 \%$ & $30 \%$ \\
\hline $\begin{array}{l}\text { 2.2.7. Aplicar nuestras estructuras gramaticales para el dominio de otras } \\
\text { lenguas. }\end{array}$ & $30 \%$ & $70 \%$ \\
\hline 2.2.8. Otras (especificar) & $10 \%$ & $90 \%$ \\
\hline
\end{tabular}

Tabla 6. Actividades gramaticales.

\section{c) Respecto a la aplicación de la enseñanza de la lengua}

En este apartado, la valoración media de los ítems es de 3,67, sobre 5 (Tabla 7). Dentro de los que tienen una valoración baja hay dos (3.5 y 3.10$)$ que tienen en común estar relacionados con el trabajo interdisciplinar en colaboración con profesores de otros departamentos. Se observa una clara correlación con las respuestas dadas al respecto en el apartado de planificación, por lo que se puede afirmar que los profesores no llevan a cabo la planificación de la competencia lingüística desde un planteamiento transversal e interdisciplinar.

Dentro de los ítems con una alta aceptación hemos de mencionar los cuatro primeros, referidos al desarrollo de las cuatro destrezas y al uso de la lengua en contexto (4,32 de media) y el ítem 3.7, relativo a la homogeneidad en la terminología gramatical. El resto de ítems tiende a una representación intermedia en su valoración. Se trata de cuestiones relacionadas 
con los elementos comunicativos no verbales, con el uso de la lengua respecto a los medios de comunicación y el respeto a la diversidad lingüística, es decir, con cuestiones relacionadas con el uso de la lengua. Los que resultan con menor preferencia son el 3.5 y el 3.10, ambos relacionados con la colaboración con profesorado que imparte otras materias diferentes.

Como conclusión a este análisis, podemos constatar que el profesorado de este estudio da gran importancia al desarrollo de dichas destrezas, por lo que declara en relación con la planificación que coincide con el resultado del bloque de aplicación. Sin embargo, sus respuestas sobre la lengua en contexto no coinciden tanto en la planificación -donde no consideran tan importante el estudio de las variedades de la lengua-, y en la aplicación -donde le dan gran importancia-. Asimismo, otra manifestación de la lengua en contexto que es la variedad lingüística, en este caso la hablada en Andalucía, tampoco es considerada tan importante por los profesores de la muestra, lo que tampoco concuerda con la preferencia por la lengua en uso.

3. Aplicación de la enseñanza de la gramática:

\begin{tabular}{|c|c|}
\hline Enunciado & Valoración \\
\hline \multicolumn{2}{|l|}{ Sobre el desarrollo de las destrezas orales y escritas } \\
\hline $\begin{array}{l}\text { 3.1. Planifico tareas para que los contenidos gramaticales trabajados se pongan } \\
\text { en acción a través de la construcción de textos orales y escritos. }\end{array}$ & 4,15 \\
\hline 3.2. Organizo tareas donde se integran las cuatro destrezas. & 4,15 \\
\hline \multicolumn{2}{|l|}{ Sobre la reflexión metalingüística y el uso de la lengua en contexto } \\
\hline $\begin{array}{l}\text { 3.3. Planifico actividades en las que los alumnos puedan reflexionar sobre la } \\
\text { lengua en diferentes contextos de uso y posteriormente ser autónomo. }\end{array}$ & 4,5 \\
\hline $\begin{array}{l}\text { 3.4. Organizo tareas en las que el alumno pueda adecuar el uso de la lengua a } \\
\text { cada situación. }\end{array}$ & 4,5 \\
\hline \multicolumn{2}{|l|}{ Sobre el planteamiento comparatista de la lengua } \\
\hline $\begin{array}{l}\text { 3.5. Promuevo y planifico proyectos interdisciplinares en equipo con el } \\
\text { profesorado que imparte otras lenguas (inglés, francés, latín...) }\end{array}$ & 2,7 \\
\hline $\begin{array}{l}\text { 3.6. Trabajo conceptos universales de las lenguas para fomentar la reflexión } \\
\text { interlingüística } \\
\text { Sobre la gramática como base del conocimiento de los elementos qu } \\
\text { comunicación }\end{array}$ & conforman la \\
\hline $\begin{array}{l}\text { 3.7. En el centro se trabaja de forma consensuada la terminología gramatical } \\
\text { para que en todos los cursos se empleen los mismos términos }\end{array}$ & 4,2 \\
\hline $\begin{array}{l}\text { 3.8. Planteo actividades en las que el alumno pueda reflexionar sobre los } \\
\text { elementos comunicativos no verbales. }\end{array}$ & 3,65 \\
\hline
\end{tabular}




\begin{tabular}{|l|c|}
\hline $\begin{array}{l}\text { Sobre la enseñanza de la gramática como instrumento para el uso de los medios de } \\
\text { comunicación y la tecnología }\end{array}$ \\
\hline $\begin{array}{l}\text { 3.9. Planifico actividades que permiten al alumno tener autonomía en el uso } \\
\text { de medios de comunicación y tecnologías de la información }\end{array}$ & 3,8 \\
\hline $\begin{array}{l}\text { 3.10. Planteamos tareas en colaboración con el departamento de nuevas } \\
\text { tecnologías de la información y la comunicación }\end{array}$ & 2,3 \\
\hline $\begin{array}{l}\text { Sobre la enseñanza de la gramática como facilitadora de la comprensión de la realidad } \\
\text { lingüística de España }\end{array}$ & 3,5 \\
\hline $\begin{array}{l}\text { 3.11. Planifico actividades que fomenten en el alumno el respeto por otras } \\
\text { lenguas. }\end{array}$ & 3,6 \\
\hline $\begin{array}{l}\text { 3.12. Hacemos actividades en las que se ayude al alumno a diferenciar el } \\
\text { contexto de uso escrito de la lengua estándar y la variedad hablada en } \\
\text { Andalucía. }\end{array}$ & \multirow{2}{|}{} \\
\hline
\end{tabular}

Tabla 7. Aplicación de la gramática.

\subsection{Respecto a la rúbrica de evaluación de los libros de texto}

En primer lugar, como evidencia general podemos decir que los libros de texto todavía no están preparados para abordar ni impulsar el cambio metodológico necesario en el estudio de la lengua. La valoración global de los libros de textos, como material para la enseñanza de la lengua desde un enfoque comunicativo, presenta un valor de 2,3 sobre 5, quedando detalladas las medias de cada ítem (Tabla 8 ). No obstante, hay que destacar que en los ítems 1.8 y 1.15 se alcanza una valoración de 5 porque son los únicos que se cumplen en todos los casos. El primero de ellos se refiere a la comprensión escrita que es esperable encontrar pues tradicionalmente se ha trabajado más que la oral, mientras que el segundo hace alusión a la uniformidad terminológica que también es esperable encontrar en un mismo libro. Los otros dos que obtienen una valoración mayor (1.9 y 1.21), se refieren a la producción escrita y al planteamiento didáctico de orden deductivo que corroboran una concepción del proceso de enseñanza de la lengua anclado en moldes tradicionales. Del resto, la mayoría están por debajo del 3, y hay un número significativo de ítems -un total de 11- que están por debajo de la valoración de 2 . Es significativo que los ítems con el valor más bajo, y por tanto con menor aparición sean los que corresponden a la transversalidad, ya sea entre áreas lingüísticas como en otras materias. Relacionándolo con los resultados del cuestionario realizado a los docentes se confirma que ni por su parte, ni por parte de los materiales docentes se facilita el aprendizaje basado en la interconexión de contenidos y en una perspectiva interdisciplinar. 


\begin{tabular}{|c|c|}
\hline Enunciado & Valoración \\
\hline 1.1. Las actividades son relevantes y significativas. & 2,6 \\
\hline 1.2. Las actividades fomentan el aprendizaje colaborativo. & 2,3 \\
\hline $\begin{array}{l}\text { 1.3. Aparecen actividades que atienden a los distintos niveles de aprendizaje } \\
\text { del alumnado. }\end{array}$ & 1,4 \\
\hline 1.4. Se proponen actividades contextualizadas a partir de textos reales. & 2,6 \\
\hline 1.5. Propone actividades de síntesis para trabajar desde el error. & 2,2 \\
\hline $\begin{array}{l}\text { 1.6. Aparecen actividades previas sobre norma y uso para la reflexión } \\
\text { lingüística. }\end{array}$ & 2,3 \\
\hline $\begin{array}{l}\text { 1.7. Aparecen actividades de síntesis sobre norma y uso para la reflexión } \\
\text { lingüística. }\end{array}$ & 1,7 \\
\hline $\begin{array}{l}\text { 1.8. Las actividades están planteadas para facilitar y promover la comprensión } \\
\text { escrita. }\end{array}$ & 5 \\
\hline 1.9. Las actividades favorecen la producción escrita. & 4,2 \\
\hline 1.10. Hay recursos audiovisuales que faciliten la comprensión oral. & 2,3 \\
\hline 1.11. Las actividades promueven la interacción oral. & 3,2 \\
\hline 1.12. Las actividades promueven un uso comunicativo de la lengua & 2,4 \\
\hline 1.13. Hay actividades que integren las cuatro destrezas. & 2,9 \\
\hline $\begin{array}{l}\text { 1.14. Las actividades están planteadas como estrategias para una tarea final de } \\
\text { creación textual }\end{array}$ & 2,2 \\
\hline 1.15. La terminología sintáctica sigue un criterio lógico y unitario. & 5 \\
\hline $\begin{array}{l}\text { 1.16. Las actividades promueven un uso de la lengua adecuado a cada } \\
\text { situación. }\end{array}$ & 2,2 \\
\hline 1.17. Hay referencias a las estructuras gramaticales de otras lenguas. & 1 \\
\hline 1.18. Las lecciones permiten o facilitan el trabajo colaborativo entre áreas. & 1,1 \\
\hline $\begin{array}{l}\text { 1.19. Hay extensión de actividades para trabajar o conectar con otros libros de } \\
\text { otras materias. }\end{array}$ & 1 \\
\hline $\begin{array}{l}\text { 1.20. Hay actividades que permiten al alumno poder comparar las estructuras } \\
\text { de diferentes lenguas. }\end{array}$ & 1 \\
\hline $\begin{array}{l}\text { 1.21. Las actividades vienen planteadas desde un enfoque deductivo } \\
\text { (planteamiento del concepto más actividades de verificación por parte } \\
\text { del alumnado). }\end{array}$ & 4,3 \\
\hline 1.22. Hay referencias a los usos pragmáticos de la lengua. & 2,3 \\
\hline 1.23. Se tratan los elementos no verbales de la comunicación. & \\
\hline $\begin{array}{l}\text { 1.24. Las actividades están planteadas para potenciar el dominio de la lengua } \\
\text { en el uso de los medios de comunicación y las tecnologías de la } \\
\text { comunicación (SMS, foros, blogs, correo electrónico, etc.). }\end{array}$ & 1,5 \\
\hline 1.25. Las actividades contienen prácticas letradas en soporte digital. & 1,5 \\
\hline $\begin{array}{l}\text { 1.26. Las actividades fomentan el conocimiento de la diversidad lingüística de } \\
\text { nuestro país en el plano morfosintáctico. }\end{array}$ & 2 \\
\hline
\end{tabular}




\begin{tabular}{|c|c|}
\hline $\begin{array}{c}\text { 1.27. Las actividades ayudan a comparar las diferentes lenguas y dialectos de } \\
\text { España desde el punto de vista morfosintáctico. }\end{array}$ & 1,6 \\
\hline $\begin{array}{c}\text { 1.28. Las actividades permiten al alumno diferenciar los contextos de uso de la } \\
\text { variedad estándar y la variedad hablada en Andalucía. }\end{array}$ & 1,5 \\
\hline
\end{tabular}

Tabla 8. Rúbrica de evaluación de libros de texto.

Para apoyar el análisis de la rúbrica hemos de hacer una consideración general acerca de la estructura de las lecciones en cada uno de los libros. Esta es muy similar ya que coinciden en la distribución de bloques de contenido: a)Comunicación, b)Estudio de la lengua, y c)Literatura. El primero de ellos -Comunicación- suele englobar las cuatro destrezas, con predominio de las escritas, y las tipologías textuales. Suele empezar con un texto, preferentemente literario, con un corpus de autores muy diverso y no ceñido a los clásicos. La presencia del texto literario sigue siendo muy frecuente, no se aprecia en general una utilización de modelos de textos de diversos ámbitos (Instituto Cervantes, 2002) que reflejen la realidad de la lengua. Pocos libros se inician con textos orales ni tampoco se utiliza mucho el recurso de las tecnologías. En el segundo bloque -Estudio de la lengua-, se integran contenidos relacionados con léxico, ortografía y morfosintaxis. Dependiendo del libro se subdividen las diferentes secciones atendiendo a esas diferentes temáticas o se engloba todo en un solo bloque llamado, dependiendo del libro, "Lengua", "Gramática" o "Estudio de la lengua". El tercer bloque -Literatura-corresponde a contenidos sobre un movimiento literario que por lo común tiene poca relación con los contenidos de la primera parte de la lección.

Dicha organización que predomina en los libros, independientemente de la editorial, coincide con los cuatro bloques que establece el currículo (Junta de Andalucía, 2007): hablar, escuchar y conversar; leer y escribir; educación literaria; conocimiento de la lengua. A pesar de que en él se declara que es una mera sugerencia de organización, las editoriales han tomado como una norma dividir las lecciones de los libros siguiendo ese patrón. Los dos primeros bloques -hablar, escuchar y conversar; leer y escribir- quedan englobados en el bloque temático "Comunicación", o bien se divide, según cada libro, en esos mismos dos bloques que describe el currículo, aunque hay casos en los que se da mayor presencia a las destrezas escritas que a las orales, continuando así con la descompensación histórica entre ambos tipos de destrezas. Se puede apreciar en la denominación del bloque "leer y escribir", cuando lo lógico sería Ilamarlo "leer, escribir, hablar y escuchar", ya que en realidad las cuatro destrezas se dan en la vida de forma simultánea (Ferrer, 2015, p. 53). Mientras, el bloque "Conocimiento de la lengua" es el reservado para lo que entendemos como gramática y que suele tener más protagonismo que los anteriores en la estructura de las unidades.

En nuestra opinión una organización así predispone a pensar que la reflexión metalingüística (poco desarrollada si el planteamiento es mecanicista) y el desarrollo de las destrezas son compartimentos estancos que no tienen vinculación aparente. Además, lleva también al alumno a pensar en el bloque gramatical como la parte del tema que hay que estudiar, lo que no le ayuda a comprender que todos los elementos que forman la comunicación forman parte de un todo. Esto hace que los profesores tengan la tendencia también a organizar los contenidos de los temas en función de esa organización a pesar de que realmente solo era una sugerencia procedente del Diseño Curricular Base y que ellos disponen de la flexibilidad suficiente para no tener que ceñirse a ello. Es un error repetido desde la LOGSE y en las posteriores leyes educativas que como vemos conlleva una división de las unidades desprovista de las explicaciones pertinentes sobre cómo integrar el uso y la reflexión lingüística (Lomas et al., 1993; 
Ruiz Bikandi, 2010). A ello hay que sumar que la fundamentación teórica de la sintaxis tiene una base diferente, anclada en modelos formales (estructuralismo y generativismo), a la del texto y el discurso, lo que dificulta una integración entre esos contenidos si no se toman en cuenta metodologías basadas en el desarrollo de la lengua en uso (Camps, 2014, p. 9). Tomar como unidad de análisis la oración frente al texto provoca una visión muy limitada del hecho lingüístico. Siguiendo el modelo de metodologías centradas en una didáctica globalizadora que parta del nivel textual (Camps, 2003; Romero Oliva et al., 2020; entre otras) se daría respuesta a esa necesaria vinculación entre los diferentes niveles lingüísticos que facilitaría la reflexión metalingüística en el alumnado.

\section{Conclusiones desde el contraste de los resultados}

A lo largo de esta investigación hemos intentado analizar los planteamientos de los docentes y de los libros de texto para encontrar una vinculación en cómo se perpetúa una forma de enseñar lengua que no es acorde con los avances del currículo y la didáctica de la lengua.

En los resultados hemos observado claramente que ni los libros de textos ni los docentes que los utilizan se muestran favorables a trabajar la lengua desde las otras áreas del currículo, mostrando ausencia de coordinación con dichas áreas, pese a que desde este último se invita a hacerlo (Fontich, 2011). Actualmente, los libros de texto no están concebidos para una enseñanza de la lengua enfocada al uso y con una perspectiva interdisciplinar (López Sánchez et al., 2018). Deben crearse materiales que faciliten el trabajo colaborativo entre áreas para fomentar la adquisición de las diferentes competencias clave y pensados para la vinculación directa de las aplicaciones de los contenidos lingüísticos a situaciones reales que traduzcan los saberes conceptuales a saberes procedimentales. A tenor de las evidencias mostradas en el apartado 3.1., se observa que tanto el libro de texto, como el profesorado no están preparados aún para un salto metodológico que beneficiaría claramente la mejora de los usos lingüísticos de los alumnos. También se evidencia que los docentes se muestran partidarios de enseñar la lengua en contexto y enfocada al desarrollo de las habilidades lingüísticas, pero hay contradicciones al dejar de lado aspectos relativos al contexto como la diversidad lingüística, los elementos extraverbales o el uso de la lengua en los medios de comunicación. También existe una clara contradicción al chocar en la aplicación con la preferencia por ejercicios enfocados al conocimiento del sistema y de naturaleza mecanicista y, por tanto, descontextualizados al tomar como referencia los modelos formales anteriores a la Lingüística del Texto que tienen como base la palabra y la oración (Garrido y Romero Oliva, 2020).

Ese tipo de libro de texto debe favorecer la interacción oral como punto de partida para la reflexión lingüística, que ayude al alumno a vincular el aprendizaje a otras situaciones comunicativas y ser progresivamente autónomo. La interacción entre alumnos y de estos con el docente permite que se lleve a cabo una construcción conjunta del conocimiento (Fontich, 2011; Milian y Ribas, 2016), ya que el diálogo constante expone al alumnado aun proceso de ida y vuelta que va desde el uso a la reflexión lingüística Especialmente necesario es ese trasvase entre las áreas dedicadas al aprendizaje de las diferentes lenguas presentes en la educación y la sociedad, en aras de formar ciudadanos capaces de adquirir la competencia en comunicación lingüística en un sentido global. Para ello es necesario fomentar la reflexión integrada sobre las diferentes lenguas, algo que todavía es una asignatura pendiente en la escuela española (Milian, 2010). Finalmente, el aprendizaje que fomente un libro de texto enfocado a una ense- 
ñanza comunicativa de las lenguas debe ser cooperativo, reforzando el papel de los alumnos como agentes sociales que interactúan gestionando sus propios procesos de aprendizaje. En este contexto, ese libro de texto debe favorecer el rol del docente como gestor del aprendizaje de sus alumnos, dejando de ser un transmisor de saber.

Desde estas páginas proponemos metodologías que vayan dirigidas al desarrollo de las habilidades lingüísticas y que tengan como foco principal el texto y el discurso (Romero Oliva et al., 2020). El enfoque por tareas (Estaire, 2009; Zanón, 1999), las propuestas desde la didáctica del texto (Álvarez Angulo, 2010; Romero Oliva, 2009) las Secuencias Didácticas para Aprender a Escribir (Camps, 2003) o las Secuencias Didácticas para aprender Gramática (Camps y Zayas, 2006), entre otras, son algunas de las vías que desde nuestra perspectiva creemos favorecedoras del cambio metodológico necesario. El libro de texto debe basarse en este tipo de enfoques para así, de la mano de los docentes, convertirse en motores del cambio necesario que haga que los alumnos pasen de saber lengua a saber utilizarla.

\section{Referencias}

Álvarez Angulo, T. (2010). Competencias básicas en escritura. Octaedro. Álvarez Angulo, T. (2013). Didáctica de la lengua para la formación de maestros. Octaedro. Alsina, R., Fortuny, J., Martí, S. y Picó, C. (2008). Lengua castellana y Literatura $4 .^{\circ}$ de ESO. Teide. Andrés, P., González, M., y Jiménez, M. (2011). Leng_o4. Aula 360 ${ }^{\circ}$. Edelvives.

Austin, J. (1962). Cómo hacer cosas con las palabras. Paidós, 1982.

Bazarra, L. y Casanova, O. (2011). Lengua castellana y Literatura $4 .^{\circ}$ de ESO. SM.

Camps, A. (2003). Secuencias didácticas para aprender a escribir. Graó.

Camps, A. (2014). Hacia una renovación de la enseñanza de la gramática. Lenguaje y Textos, 40, 7-18. https://bit.ly/3yzixbF

Camps, A. (2017). Enseñar gramática. En A. Camps y T. Ribas (Coords.), El verbo y su enseñanza. Hacia un modelo de enseñanza de la gramática basado en la actividad reflexiva (pp. 17-74). Octaedro.

Camps, A. y Zayas, F. (2006). Secuencias didácticas para aprender gramática. Graó.

Cassany, D., Luna, M. y Sanz, G. (1994). Enseñar lengua. Graó.

Castellà, J. M. (1994). ¿Qué gramática para la escuela? Sobre árboles, gramáticas y otras formas de andarse por las ramas. Textos de Didáctica de la Lengua y la Literatura, 2, 15-23. https://bit.ly/3sjmGzE

Chevallard, Y. (1985). La transposition didactique, du savoir savantau savoir enseigné. Le pensée sauvage.

Escolano, A. (1997). El libro escolar: perspectivas históricas. En L. Arranz Márquez (Coord.), El libro de texto. Materiales didácticos. Actas del $5^{\circ}$ congreso (pp. 37-50). Universidad Complutense.

Estaire, S. (2009). El aprendizaje de lenguas mediante tareas: de la programación al aula. Edinumen.

Ferrer, M. (2015). La función del libro de texto en la clase de lengua. Textos de Didáctica de la Lengua y la Literatura, 69, 51-59. http://hdl.handle.net/11162/115425

Fontich, X. (2011). La enseñanza de la gramática en primaria y secundaria: algunas reflexiones y propuestas. Da Investigação às Práticas, 1(2), 38-57. https://doi.org/10.25757/invep.v1i2.61

Fontich, X. (2013). La gramática de la primera lengua en la escuela: reflexiones sobre su enseñanzaaprendizaje y sobre el contenido gramatical escolar. Bellaterra Journal of Teaching \& Learning Language \& Literature, 6(3), 1-19. https://doi.org/10.5565/rev/jtl3.531

Garrido, F. y Romero Oliva, M.F. (2020). Enseñanza de la gramática y lingüística del texto: una secuencia didáctica para el aula de Educación Secundaria. Sabir, International Bulletin of Applied 
Linguistics, 1(2). https://doi.org/10.25115/ibal.v1i2.3501

Gómez, J., Lajo, J., Toboso, J. y Vidorreta, C. (2011). Lengua castellana y Literatura. $4 .^{\circ}$ de ESO Andalucía. Bruño.

González, J. A. (Coord.), Ariza, J., Coca, I., Hoster, B. y Ruiz, A. (2008). Lengua castellana y Literatura. $4^{\circ}$ de ESO (Andalucía). Algaida.

González Nieto, L. (2001). Teoría lingüística y enseñanza de la lengua (Lingüística para profesores). Cátedra.

Gutiérrez, A. y Gaviño, V. (2020). La marca de la Real Academia Española en los manuales de enseñanza de la lengua para la Educación Secundaria: el caso de la oración. Educaçao \& Formaçao, 5(3). https://doi.org/10.25053/redufor.v5i15set/dez.2891

Jiménez Fernández, R. (2011). La gramática en los manuales de la eso: Actividades sobre morfosintaxis. Cauce. Revista internacional de Filología, Comunicación y sus Didácticas. 34-35, 231-255. http://hdl.handle.net/11441/22082

Johansson, M. (1988). El libro de texto imposible. En T. Rönström (Ed.), Libros escolares 1-3. (pp. 22-24). Estocolmo.

Johnsen, E. B. (1996). Libros de texto en el calidoscopio: estudio crítico de la literatura y la investigación sobre los textos escolares. Pomares-Corredor.

Juan, E. (dir.) et al. (2011). Lengua y Literatura $4 .^{\circ}$ de ESO. Santillana-Grazalema.

Junta de Andalucía (2007). Orden de 10 de agosto de 2007, por la que se desarrolla el currículo correspondiente a la Educación Secundaria Obligatoria en Andalucía. Sevilla. Boletín Oficial de la Junta de Andalucía. 171(30 de agosto de 2007), 23-65.

Instituto Cervantes (2002). Marco Común Europeo de Referencia para las Lenguas: Aprendizaje, Enseñanza, Evaluación. Recuperado de: http://cvc.cervantes.es/obref/marco.

Ley de Instrucción Pública (1857). Gaceta de Madrid, $n^{\circ} 1710$, 10 de septiembre de 1857 1-3.

Ley General de Educación (1970). Boletín Oficial del Estado no 187, 4 de agosto de 1970, 12525-12546.

Ley de Ordenación General del Sistema Educativo (1990). Boletín Oficial del Estado $n^{\circ} 238,3$ de octubre de 1990, 28927-28942.

Ley Orgánica de Calidad de la Educación (2002). Boletín Oficial del Estado $n^{\circ}$ 307, 23 de diciembre de 2002, 45188-45220.

Ley Orgánica de Educación (2006). Boletín Oficial del Estado no 106, 4 de mayo de 20026, 17158-17207.

Ley Orgánica para la Mejora de la Calidad de la Educación (2013). Boletín Oficial del Estado $n^{\circ} 295$, 9 de diciembre de 2013, 97858-97921.

Lomas, C., Osoro, A. y Tusón, A. (1993). Ciencias del lenguaje, competencia comunicativa y enseñanza de la lengua. Paidós.

López Sánchez, F. J., García, F. J. y Travé, G. (2018). La enseñanza sobre el medio y los libros de texto en Andalucía: un análisis de contenido y de concepciones del profesorado. Revista Complutense de Educación, 29(2), 539-557. https://doi.org/10.5209/RCED.53450

López, F. y Serrano, S. (2008). Lengua castellana y Literatura $4^{\circ}$ de Secundaria. Proyecto Ánfora. Oxford Educación.

Martínez Montes, G. T., López Villalva, M.A. y Juárez, M.G. (2015). El enfoque comunicativo (pp. 75-94). En C. Lomas (Coord.), Fundamentos para una enseñanza comunicativa del lenguaje. Graó.

Martín Vegas, R.A. (2009). Manual de Didáctica de la Lengua y la Literatura. Síntesis.

McMillan, J. H. y Schumacher, S. (2005). Investigación educativa. Pearson Educación.

Milian, M. (2010). La gramática en los manuales de lengua: ¿qué actividades se proponen a los alumnos?. En T. Ribas (Coord.), Libros de texto y enseñanza de la gramática (pp. 155-172). Graó.

Milian, M. y Ribas, T. (2016). La reflexión metalingüística y la gramática. En J. Palou y M. Fons (Coords.), Didáctica de la Lengua y la Literatura en Educación Primaria (pp. 213-226). Síntesis. 
Navarro, E. y Reina, A. (2011). Lengua castellana y Literatura $4^{\circ}$ de ESO. Casals.

Prado, J. (2011). Didáctica de la lengua y la literatura para educar en el siglo XXI. La Muralla.

Romero Oliva, M. F. (2009). Acceso a las competencias básicas desde la lecto-escritura. Tabanque Revista Pedagógica, 22, 191-204. http://uvadoc.uva.es/handle/10324/8928

Romero Oliva, M. F., Jiménez Fernández, R. y Trigo, E. (2020). Donde habita el olvido: recuperando el lugar de la gramática en las aulas. Lublin Studies in Modern Languages and Literature, 44(3), 1-15. http://dx.doi.org/10.17951/lsmll.2020.44.3.117-129

Ruiz Bikandi, U. (2010). El conocimiento de la lengua en el Decreto de Enseñanzas mínimas de 2007. Una revisión crítica. En T. Ribas (Coord.), Libros de texto y enseñanza de la gramática (pp. 33-54). Graó.

VVAA (2008). Lengua y Literatura $4^{\circ}$ de ESO. Guadiel-Grupo Edebé.

Zanón, J. (1999). La enseñanza del español mediante tareas. Edinumen.

Zayas, F. (2006). Trabajamos la oración. En A. Camps y F. Zayas (Coords.), Secuencias didácticas para aprender gramática (pp. 147-160). Graó.

Zayas, F. (2011). El lugar de la gramática en la enseñanza de la lengua. En U. Ruiz Bikandi (Coord.), Lengua castellana y Literatura. Investigación, innovación y buenas prácticas (pp. 91-106). Graó. 